\title{
Aligned magnetic field flow of Williamson fluid over a stretching sheet with convective boundary condition
}

\author{
$N S$ Arifin ${ }^{1}, S M Z_{\text {Zokri }}{ }^{1}, A R M \mathrm{Kasim}^{1,{ }^{*}}, M Z \mathrm{Salleh}^{1}$, and $N F$ Mohammad $^{2}$ \\ ${ }^{1}$ Applied \& Industrial Mathematics Research Group, Faculty of Industrial Sciences \& Technology, \\ Universiti Malaysia Pahang, 26300 Gambang, Kuantan, Pahang, Malaysia \\ ${ }^{2}$ Department of Computational and Theoretical Sciences, Kulliyyah of Sciences, International Islamic \\ University Malaysia, 25200 Kuantan, Pahang, Malaysia
}

\begin{abstract}
The analysis on mathematical model of Williamson fluid on the convective boundary layer flow and heat transfer over a horizontal stretching sheet has been carried out. The model is constructed by introducing the aligned magnetic field on the flow region under the case of convective boundary conditions which is implies the fluid heated from the stretching surface. The numerical method of Runge-Kutta Fehlberg (RKF45) is employed to the ordinary differential equations after being transformed from the governing equations by using suitable similarity transformation. The solutions of several influencing parameters on the velocity and temperature profiles are discussed and displayed in graphical form. The results of this study indicate that the increasing magnetic field parameter decreases the fluid velocity which suppressed the fluid motion.
\end{abstract}

\section{Introduction}

Convective heat transfer and fluid flow over a stretching sheet has been one of the major interesting research subjects either from the theoretical perspectives and also experimental practices because of the rapid development in the industrial manufacturing process for metal and polymer sheet. The quality of the sheet production is solely depended on the types of fluid and heat transfer diffusivity with certain particular effects that might prevails the whole process. To cover such dimension, numerous studies have concentrated on Newtonian and non-Newtonian fluid together with the effect of magnetic field, viscous dissipation, stretching parameter, different thermal boundary conditions and etc.

The Newtonian fluid flow with constant wall temperature (CWT) and constant heat flux (CHF) boundary conditions are applied on the surface of several geometries have been attempted, such as the works of [1-4] for the heat transfer analysis. However, it is seemed relevant assumption for the heated body surface resulting from the convection of hot fluid which has been analyzed by Aziz [5]. This thermal boundary condition or generally addressed as convective boundary condition (CBC) is an alternative choice for researches besides the common isothermal and isoflux boundary conditions. Along with this, there is

* Corresponding author: rahmanmohd@ump.edu.my 
increasing concern for investigating the flow problems with different cases [6-10]. Following these, it is motivate us to analyze the heat transfer problem with $\mathrm{CBC}$ by including the aligned magnetic field for the Williamson fluid on the flow region. This paper begins by formulating the governing equations of fluid model which then are reduced into appropriate form. The numerical results for velocity and temperature profiles are obtained using Runge-Kutta Fehlberg (RKF45) method.

\section{Mathematical formulation}

Consider a steady, incompressible Williamson fluid over a horizontal stretching sheet with uniform velocity $u_{w}(x)$ with an aligned magnetic field is applied on the flow as illustrated in fig. 1 . The bottom of the sheet is heated by the convective boundary condition.

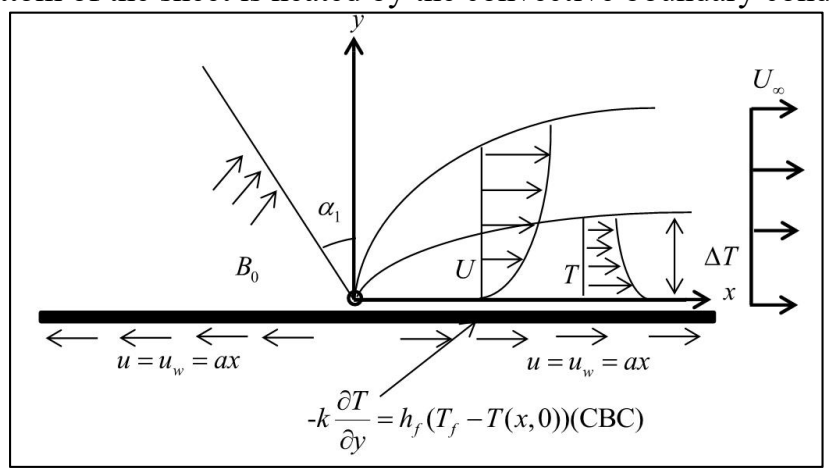

Fig. 1. Flow configuration.

Under the boundary layer and Boussinesq approximations, the governing equations for the flow model are (see [11-13])

$$
\begin{gathered}
\frac{\partial u}{\partial x}+\frac{\partial v}{\partial y}=0 \\
u \frac{\partial u}{\partial x}+v \frac{\partial u}{\partial y}=v\left(\frac{\partial^{2} u}{\partial y^{2}}\right)+\sqrt{2} v \Gamma \frac{\partial u}{\partial y} \frac{\partial^{2} u}{\partial y^{2}}-\frac{\sigma}{\rho} u B_{0}^{2} \sin ^{2} \alpha_{1} \\
u \frac{\partial T}{\partial x}+v \frac{\partial T}{\partial y}=\alpha\left(\frac{\partial^{2} T}{\partial y^{2}}\right)
\end{gathered}
$$

where $(u, v)$ are the velocity components of the fluid along $x$ and $y$ axes, respectively. $T$ is the fluid temperature, $v$ is the kinematic viscosity, $\Gamma>0$ is the time constant, $\rho$ is the fluid density, $\alpha_{1}$ is the aligned angle, $B_{0}$ is the magnetic-field strength, $\sigma$ is the electrical conductivity and $\alpha$ is the thermal diffusivity. (3)

The following boundary conditions are introduced to the stated governing equations (1)-

$$
\begin{gathered}
u=u_{w}(x)=a x, v=0,-k \frac{\partial T}{\partial y}=h_{f}\left(T_{f}-T(x, 0)\right) \quad \text { at } y=0 \\
u \rightarrow 0, T \rightarrow T_{\infty}, \quad \text { as } y \rightarrow \infty
\end{gathered}
$$

where $a$ is positive constant, $h_{f}$ is heat transfer coefficient, $k$ is the thermal conductivity, $T_{f}$ is the hot fluid temperature and $T_{\infty}$ is the ambient temperature. 
The similarity variable and functions are defined as follows,

$$
u=a x f^{\prime}(\eta), \quad v=-(a v)^{1 / 2} f(\eta), \quad \eta=\left(\frac{a}{v}\right)^{1 / 2} y, \quad \theta(\eta)=\frac{T-T_{\infty}}{T_{f}-T_{\infty}},
$$

where the stream function $\psi$ is generally denoted as $u=\partial \psi / \partial y$ and $v=-\partial \psi / \partial x$. The resulting dimensionless ordinary differential equations (1)-(3) can be expressed as

$$
\begin{gathered}
f^{\prime \prime \prime}(\eta)+f(\eta) f^{\prime \prime}(\eta)-\left(f^{\prime}(\eta)\right)^{2}+\lambda f^{\prime \prime}(\eta) f^{\prime \prime \prime}(\eta)-M \sin ^{2} \alpha_{1} f^{\prime}(\eta)=0, \\
\frac{1}{\operatorname{Pr}} \theta^{\prime \prime}(\eta)+f(\eta) \theta^{\prime}(\eta)=0,
\end{gathered}
$$

and the boundary conditions (4) are reduced to

$$
f(0)=0, f^{\prime}(0)=1, \quad \theta^{\prime}(0)=-\gamma(1-\theta(0)) \text { at } \eta=0
$$

where a prime (') denotes differentiation with respect to $\eta . M=\sigma B_{0}^{2} / \rho a$ is the magnetic field parameter, $\lambda=\sqrt{2 a^{3} / v} \Gamma x$ is the Willliamson parameter, $\operatorname{Pr}=\mu c_{p} / k$ is the Prandtl number, and $\gamma=\left(h_{f} / k\right)(v / a)^{1 / 2}$ is the conjugate parameter for CBC.

The physical quantities of the skin friction coefficient $C_{f}$ and the local Nusselt number $N u_{x}$ are defined by

$$
C_{f}=\frac{\tau_{w}}{(1 / 2) \rho U^{2}(x)}, \quad N u_{x}=\frac{x q_{w}}{k\left(T_{w}-T_{\infty}\right)},
$$

where $\tau_{w}=\mu(\partial u / \partial y)_{y=0}$ and $q_{w}=-k(\partial T / \partial y)_{y=0}$ are the rate of heat transfer and surface heat flux respectively. Introduce (5) into equation (9), yield

$$
C_{f} \operatorname{Re}_{x}^{1 / 2}=f^{\prime \prime}(0)+\frac{\lambda}{2}\left(f^{\prime \prime}(0)\right)^{2}, \quad N u_{x} \operatorname{Re}_{x}^{-1 / 2}=\gamma\left(\frac{1}{\theta(0)}-1\right)
$$

where $\operatorname{Re}_{x}=\left(a x^{2} / v\right)$ is the Reynolds number.

\section{Numerical solution}

The numerical solutions of the dimensionless ordinary differential equations (6)-(8) are solved using Runge-Kutta Fehlberg (RKF45) method, whereby the algorithm is built in Maple software. The efficiency of the method has been approved by [5]. The finite value of maximum boundary layer thickness $\eta_{\infty}$ is selected in such a way that the asymptotic boundary conditions (8) are successfully achieved. In accordance to this, $\eta_{\infty}=7$ is set for the whole calculation process together with the controlling parameters involved.

\section{Results and discussion}

The present problem reduces to the viscous fluid $(\lambda=0)$ flow without the effect of aligned magnetic field $\left(M=\alpha_{1}=0\right)$ for isothermal condition $(\gamma \rightarrow \infty)$. This case is fortunately similar with that flow problems of Goyal and Bhargava [14] and Freidoonimehr and Rashidi [15] by limiting some controlling parameters. For the sake of accurateness, the numerical data herein is compared with the data obtained from numerical method of finite 
element method (FEM) and also analytical method of homotopy analysis method (HAM) that has been conducted in both researches respectively. It is revealed in table 1 that the present numerical values of $-\theta^{\prime}(0)$ are in a great agreement with the previous works. Therefore, the numerical results presented here are considered accurate.

A brief discussion on the fluid flow and heat transfer with the influencing parameters of aligned angle $\alpha_{1}$, magnetic field parameter ${ }_{M}$, Williamson parameter $\lambda$, Prandtl number Pr and conjugate parameter $\gamma$ for $\mathrm{CBC}$ has been made based on the plotted graph. The numerical computation is accomplished by fixing the parameters value to $\alpha_{1}=\pi / 3, M=1, \lambda=0.2, \operatorname{Pr}=10, \gamma=0.5$.

Table 1. Comparison results for $-\theta^{\prime}(0)$ when $\lambda=M=\alpha_{1}=0$ and $\gamma \rightarrow \infty$.

\begin{tabular}{|c|c|c|c|}
\hline $\operatorname{Pr}$ & Goyal and Bhargava[14] & Freidoonimehr and Rashidi [15] & Present \\
\hline 0.20 & 0.1691 & 0.1691 & 0.1691 \\
\hline 0.70 & 0.4539 & 0.4539 & 0.4539 \\
\hline 2.0 & 0.9113 & 0.9114 & 0.9113 \\
\hline 7.0 & 1.8954 & 1.8954 & 1.8954 \\
\hline
\end{tabular}

The variation values of magnetic field parameter $M$ on the velocity profile are shown in fig.2. It is observed from the figure that the fluid velocity profile decreases with an increase in $M$. The general explanation for this phenomenon could be that there exist a drag forces on the surface sheet produced by the increment of Lorentz force. On top of that, since the Williamson fluid is non-Newtonian fluid then the forces could be greater which reduces the fluid motion.

Fig. 3 illustrates the decreasing trend of temperature profile with conjugate parameter $\gamma$ for CBC. The temperature profile increases with $\gamma$ and it is also revealed in the figure that the surface temperature $\theta(0)$ approaching one for higher value of $\gamma$ which coincide with the commonly used boundary condition, CWT. It is interesting to note that the present problem could also turned to flow problem of Williamson fluid with CWT if $\gamma \rightarrow \infty$.

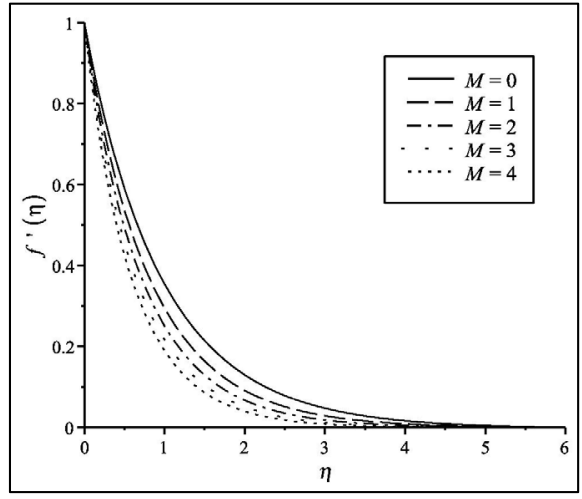

Fig. 2. Effect of $M$ on velocity profile.

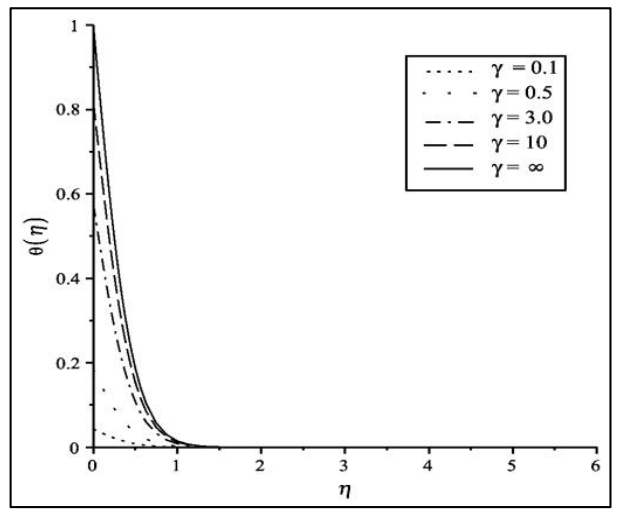

Fig. 3. Effect of $\gamma$ on temperature profile.

\section{Conclusion}


The mathematical formulation of Williamson fluid flow over a heated stretching sheet induced by convective boundary condition with the influence of aligned magnetic field on the flow region is numerically solved. The Runge-Kutta Fehlberg (RKF45) method is utilized to solve the dimensionless system of ordinary differential equations and the results obtained is interpret in the graphical form. From the present paper, it is concluded that the increasing magnetic field parameter decreases the boundary layer thickness. Conversely, the thermal boundary layer thickness enhances with conjugate parameter for $\mathrm{CBC}$.

\section{Acknowledgments}

The authors gratefully acknowledge the financial support received from Universiti Malaysia Pahang for (PGRS170397, RDU 160330, RDU 170328 \& RDU 141306).

\section{References}

1. Tsou F, Sparrow E and Goldstein R J 1967 Int J Heat Mass Transfer 10 219-235

2. Crane L J 1970 J Appl Math Phys (ZAMP) 21 645-647

3. Gupta P and Gupta A 1977 Can J Chem Eng 55 744-746

4. Shu J-J and Pop I 1998 Int J Heat Fluid Flow 19 79-84

5. Aziz A 2009 Commun Nonlinear Sci Numer Simulat 14 1064-1068

6. Rahman M and Eltayeb I 2013 Meccanica 48 601-615

7. Kasim A R M, Jiann L Y, Rawi N A, Ali A and Shafie S 2015 Defect and Diffusion Forum 362 67-75

8. Mohamed M K A, Salleh M Z, Ishak A and Pop I 2015 Eur Phys J Plus 130 1-9

9. Anwar M I, Shafie S, Kasim A R M and Salleh M Z 2016 Heat Transf Res 47 797-816

10. Arifin N S, Zokri S M, Aziz L A, Kasim A R M, Salleh M Z and Mohammad N F 2017 AIP Conf. Proc. vol 1842 p 030006

11. Makinde O D and Aziz A 2011 Int J Therm Sci 50 1326-1332

12. Salleh M Z, Nazar R and Pop I 2010 J Taiwan Inst Chem Eng 41 651-655

13. Khan NA and Khan H 2014 Nonlinear Eng 3 107-115

14. Goyal M and Bhargava R 2014 Appl Nanosci 4 761-767

15. Freidoonimehr N, Rashidi M M, Momenpour M H and Rashidi S 2017 Int J Biomath 101750008 